DESY 99-140

HLRZ1999_42

\title{
On the eta-invariant in the four dimensional chiral $U(1)$ theory
}

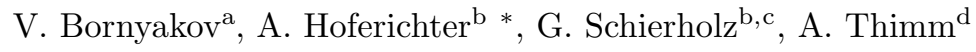 \\ ${ }^{\mathrm{a}}$ Institute for High Energy Physics IHEP, 142284 Protvino, Russia \\ ${ }^{\mathrm{b}}$ Deutsches Elektronen-Synchrotron DESY and NIC, 15735 Zeuthen, Germany \\ ${ }^{\mathrm{c}}$ Deutsches Elektronen-Synchrotron DESY, 22603 Hamburg, Germany \\ dinstitut für Theoretische Physik, Freie Universität Berlin, 14295 Berlin, Germany
}

The imaginary part of the effective action is investigated in the $4 \mathrm{D}$ chiral $\mathrm{U}(1)$ theory using the CFA.

\section{INTRODUCTION}

In the continuum the relation between chiral gauge theories in even dimensions $D$ and their vectorial counterparts is known since many years. For an external gauge field $A$ in the topologically trivial sector, the chiral fermionic effective action $W[A]$ is given by円[1] :

$$
\begin{aligned}
& \operatorname{Re}\left(W[A]-W\left[A_{0}\right]\right)=\frac{1}{2}\left(W_{\mathrm{V}}[A]-W_{\mathrm{V}}\left[A_{0}\right]\right) \\
& \operatorname{Im}\left(W[A]-W\left[A_{0}\right]\right)=\pi \eta+2 \pi Q_{5}^{0}\left(A_{t}\right)
\end{aligned}
$$

where $A_{t}=(1-t) A_{0}+t A, t \in[0,1]$, interpolates between some initial configuration $A_{0}$ and $A$ in the same topological sector and $W_{\mathrm{V}}$ denotes the effective action for the associated vector theory. The imaginary part, which conveys the chiral nature of the theory, is given by the eta-invariant $\eta$ [2] and the Chern-Simons form $Q_{5}^{0}$. The latter encodes the anomaly $\delta_{g} W[A] \propto \mathrm{i} \delta_{g} Q_{5}^{0}, \delta_{g}$ being the variation w.r.t. a gauge transformation $g$. In the anomaly free model the imaginary part is given by the eta-invariant alone, manifesting the key rôle of $\eta$ in the understanding of chiral gauge theories. Since $\eta$ is defined as the spectral asymmetry of an 'extended' Dirac operator, its presence indicates a $D+1$ dimensional underlying problem.

Despite the recent progress in constructing chiral gauge theories on the lattice [3], there seems

\footnotetext{
*Talk given by A. Hoferichter.

${ }^{2} \mathrm{Up}$ to local counter terms for the real part.
}

to be no easily manageable expression for $\eta$ at finite lattice spacing $a$. Within a 5-dimensional approach a lattice definition of the eta-invariant (and $Q_{5}^{0}$ ) has been given in [4] and shown, that in the classical continuum limit the lattice expressions transform to the known continuum expressions. From the point of view of a practical implementation of chiral gauge theories on the lattice it is desirable to control the imaginary part of the resulting chiral effective action. As a first step towards this goal we investigate the effective action of the $4 \mathrm{D}$ lattice chiral $\mathrm{U}(1)$ theory in order to extract information on the eta-invariant.

\section{LATTICE MODEL AND STRATEGY}

In matrix notation with all indices suppressed the fermionic action of the model under consideration is given by

$$
S_{F}[\bar{\psi}, \psi, U]=\bar{\psi} \mathbb{M}[U] \psi
$$

where $U$ is an external field with (compact) link variables $U_{\mu}(x) \in \mathrm{U}(1)$ and $\mathbb{M}[U]=\not D[U]+$ $\mathbb{W}[U]$. The Dirac part is written as

$\not D=\not D P_{R}+\not D P_{L}=\left(\begin{array}{cc}0 & D_{L} \\ D_{R} & 0\end{array}\right)$

with $P_{R, L}=\frac{1}{2}\left(1 \pm \gamma_{5}\right)$, and the Wilson term,

$\mathbb{W}=\left(\begin{array}{cc}W_{L R} & 0 \\ 0 & W_{R L}\end{array}\right)$

${ }^{3}$ We use standard lattice notation for one flavor. 
may in principle depend also on $U$. Here we will consider the so-called ungauged Wilson term only. In this case there are no counter terms for the imaginary part of the effective action [5]. Explicitely, we consider

$\mathbb{W}=-\frac{1}{2}\left(\begin{array}{cc}\partial^{b} \partial^{f} & 0 \\ 0 & \partial^{f} \partial^{b}\end{array}\right)$

where $\partial^{f, b}$ denotes the lattice forward and backward derivative, respectively. By choosing the right-handed component of the field $U_{R}$ to be trivial, we obtain a chiral U(1) model with $D_{R}=$ $\partial_{R}$. In this study, we restrict ourselves to the discussion of the imaginary part of the fermionic effective action

$$
\operatorname{Im} W=\operatorname{Im}(-\ln \operatorname{det} \mathbb{M}),
$$

which we evaluate in the continuum fermion approach (CFA) (e.g. [6.5.7]) by the following steps.

(1) interpolate the lattice gauge configuration $U^{a}$ on the original lattice with spacing $a$ to a continuum gauge field $A$ by a suitable procedure (e.g. 8) : $U^{a} \longrightarrow A$

(2) in order to avoid infinities, project back $A$ to a lattice configuration with finer spacing $a_{f}=m^{-1} a, m>1: A \longrightarrow U^{a_{f}}$

(3) consider $\lim _{a_{f} \rightarrow 0} \operatorname{Im} W\left[U^{a_{f}}\right]$, with $a$ fixed, to evaluate the fermionic effective action for the continuum fermions

To carry out the full program, eventually the limit $a \rightarrow 0$ has to be taken, which we will not perform here. In practice, steps (1) and (2) are combined without making the 'detour' via the continuum gauge field, such that one interpolates $U^{a} \rightarrow U^{a_{f}}$ directly. We make use of the remaining freedom in the interpolation procedure by introducing displacement vectors w.r.t. the origins of the original and finer lattices [9]. Thus, for one configuration $U^{a}$ on the original lattice we are able to obtain a set of configurations $\left\{U^{a_{f}}\right\}$. Corresponding distributions of $\operatorname{Im} W\left[U^{a_{f}}\right]$ are used to define a variance. In the $a_{f} \rightarrow 0$ limit the results should become independent of the details of interpolation, leading to narrower distributions for smaller $a_{f}$. To compute $\operatorname{det} \mathbb{M}\left[U^{a_{f}}\right]$ we apply a non-Hermitean Lanczos procedure, which has been modified for numerical stability with complete re-orthogonalization. In order to derive the information about the anomaly free model, we impose the anomaly cancellation condition

$$
\sum_{f=1}^{N} \varepsilon_{f} c_{f}^{3}=0,
$$

in an additional step. The sum extends over the $N$ different flavors with chiralities $\varepsilon_{f}$ and fermion charges $c_{f}$. After anomaly cancellation, we adopt the continuum notation by setting

$$
\lim _{a_{f} \rightarrow 0} \operatorname{Im} W\left[U^{a_{f}}\right] \stackrel{!}{=} \pi \eta ; \quad a=\text { const },
$$

which formally provides us with the notion of a lattice eta-invariant. Lattice artefacts and gauge invariance breaking effects are expected to vanish at least as $O\left(a_{f} / a\right)$ [5. [7].

\section{FIRST RESULTS}

We considered three original $L^{4}=3^{4}$ gauge configurations denoted by $U_{1,2,3}$ interpolated to lattices sizes up to $8^{4}$ as depicted schematically below:

$$
\begin{aligned}
& \{U\}^{L=3} \\
& \text { random displacements of origin } \\
& \begin{array}{ccccc}
\Downarrow & \Downarrow & \Downarrow & \Downarrow & \Downarrow \\
\{U\}^{L=4} & \{U\}^{L=5} & \{U\}^{L=6} & \{U\}^{L=7} & \{U\}^{L=8}
\end{array} .
\end{aligned}
$$

Fig.1 displays the distribution of $\operatorname{Im} W$ for the case of right (left) handed fermions and after anomaly cancellation. The original configuration $U_{1}$ has been chosen randomly, with the constraint $\left|\theta_{\mu}(x)\right|<\frac{\pi}{5}$ on the link angles $\theta_{\mu}(x)$. Although the distribution for the $\mathrm{c}=2$ case has a large variance, after anomaly cancellation the distribution is sharply peaked about some central value. This is possible since the anomaly is canceled event by event, and the error bars in Fig.2, should not be confused with Monte Carlo errors, but are an estimation of lattice errors. The $a_{f}$ dependence of

\footnotetext{
$\overline{{ }^{4} \text { We set } \mathrm{a}=1 \text { from now }}$ on and drop the superscript $a_{f}$ on interpolated configurations.
} 


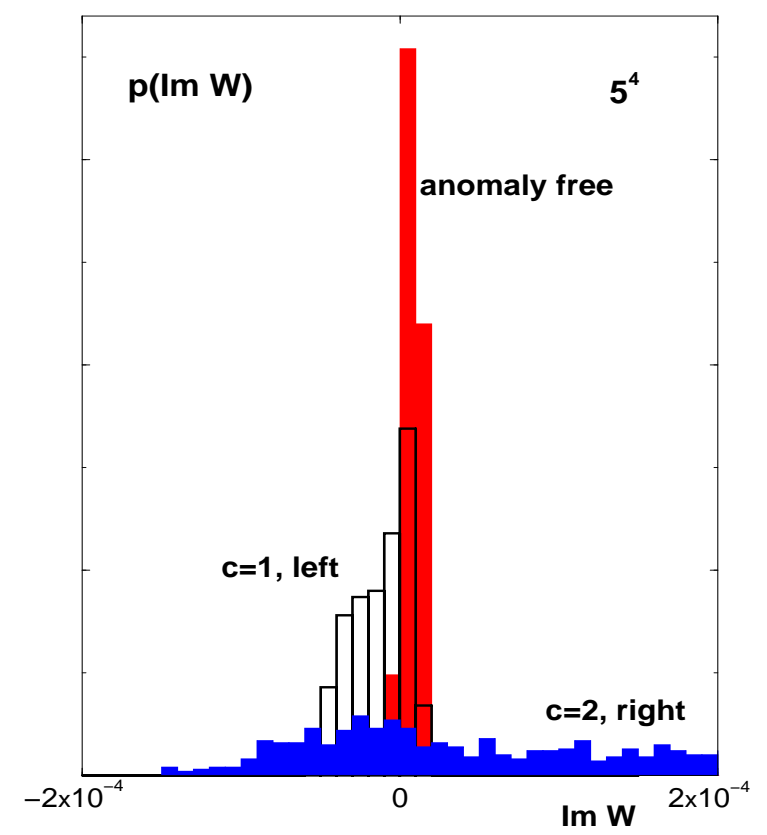

Figure 1. Distribution of $\operatorname{Im} W$ for right (left) handed fermions with given charges and for the anomaly free model for $U_{1}$ interpolated to $5^{4}$.

$\operatorname{Im} W$ for $U_{1}$ is shown in Fig. 2. For the two finest lattices we do not display error bars, since we could not evaluate the effective action for enough displacement vectors yet. The observation of Fig. 11 continues in the case of finer lattices. In the extreme case, $\operatorname{Im} W$ (including the anomaly) is about two orders of magnitude larger than the result for the anomaly free model. For the configurations $U_{k}, k=2,3$, with $\left|\theta_{\mu}(x)\right|<\frac{\pi}{6}, \frac{\pi}{8}$, respectively, we find similarly small values of $\operatorname{Im} W$ in the anomaly free case.

\section{SUMMARY}

After anomaly cancellation we find $|\operatorname{Im} W|<$ $2 \cdot 10^{-5}$ for the given configurations. Hence, in these cases, $\eta$ seems to be very small, although it can take any value mod $2 \mathbb{Z}$. The results encourage further investigation towards a dynamical simulation of a $4 \mathrm{D}$ chiral model.

\section{ACKNOWLEDGMENTS}

This work has been partially supported by the INTAS 96-370 grant. V.B. acknowledges support from RFBR 99-01230a grant. The calculations have been partly done on the T3E at ZIB, and we thank H. Stüben for technical support. Thanks go to K. Jansen (especially for pointing out the problem of counter terms for $\operatorname{Im} W[U]$ ) and to B. Andreas for discussion.

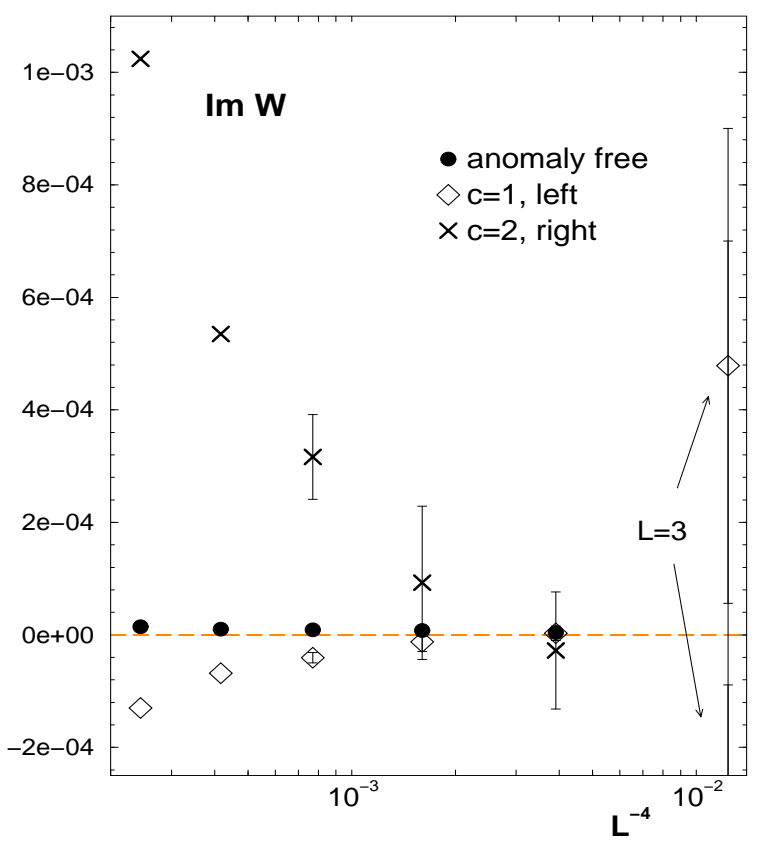

Figure 2. $\operatorname{Im} W$ vs. $L^{-4}$ for different charges and chiralities. The original configuration is $U_{1}$.

\section{REFERENCES}

1. L. Alvarez-Gaumé, S. Della Pietra, V. Della Pietra, Phys. Lett. 166B, 177 (1986).

2. M.F. Atiyah, V.K. Patodi, I.M. Singer, Math. Proc. Camb. Phil. Soc. 77, 43 (1975).

3. Talks by M. Lüscher and H. Neuberger, this volume.

4. T. Aoyama, Y. Kikukawa, hep-lat/9905003 and this volume.

5. G.T. Bodwin, Phys. Rev. D54, 6497 (1996).

6. M. Göckeler, G. Schierholz, Nucl. Phys. B (Proc. Suppl.) 29B,C 114 (1992).

7. P. Hernández, R. Sundrum, Nucl. Phys. B455, 287 (1995).

8. M. Göckeler, A.S. Kronfeld, G. Schierholz, U.-J. Wiese, Nucl. Phys. B404, 287 (1993).

9. Talk by A. Thimm, this conference. 